\title{
Demo Abstract: Supporting Heterogeneous IoT Traffic using the IEEE 802.11ah Restricted Access Window
}

\author{
Serena Santi \\ serena.santi@uantwerpen.be \\ University of Antwerp - imec \\ Belgium \\ Eli De Poorter \\ eli.depoorter@ugent.be \\ Ghent University - imec \\ Belgium
}

\author{
Amina Šljivo \\ amina.sljivo@ugent.be \\ Ghent University - imec \\ Belgium \\ Jeroen Hoebeke \\ jeroen.hoebeke@ugent.be \\ Ghent University - imec \\ Belgium
}

\author{
Le Tian \\ le.tian@uantwerpen.be \\ University of Antwerp - imec \\ Belgium \\ Jeroen Famaey \\ jeroen.famaey@uantwerpen.be \\ University of Antwerp - imec \\ Belgium
}

\begin{abstract}
IEEE $802.11 \mathrm{ah}$ is a new Wi-Fi standard operating on unlicensed sub-GHz frequencies. It aims to provide long-range connectivity to Internet of Things (IoT) devices. The IEEE 802.11ah restricted access window (RAW) mechanism promises to increase throughput and energy efficiency in dense deployments by dividing stations into different RAW groups and allowing only one group to access the channel at a time. In this demo, we demonstrate the ability of the RAW mechanism to support a large number of densely deployed IoT stations with heterogeneous traffic requirements. Differentiated Quality of Service (QoS) is offered to a small set of high-throughput wireless cameras that coexist with thousands of best-effort sensor monitoring stations. The results are visualized in near real-time using our own developed IEEE 802.11ah visualizer running on top of the ns-3 event-based network simulator.
\end{abstract}

\section{CCS CONCEPTS}

- Networks $\rightarrow$ Network performance evaluation; Network algorithms; Network management; • Sensor networks;

\section{KEYWORDS}

IEEE 802.11ah, restricted access network (RAW), ns-3, IoT, Quality of Service

\section{ACM Reference Format:}

Serena Santi, Amina Šljivo, Le Tian, Eli De Poorter, Jeroen Hoebeke, and Jeroen Famaey. 2017. Demo Abstract: Supporting Heterogeneous IoT Traffic using the IEEE 802.11ah Restricted Access Window. In Proceedings of 15th ACM Conference on Embedded Networked Sensor Systems (SenSys'17). ACM, New York, NY, USA, 2 pages. https://doi.org/10.1145/3131672.3136956

Permission to make digital or hard copies of all or part of this work for personal or classroom use is granted without fee provided that copies are not made or distributed for profit or commercial advantage and that copies bear this notice and the full citation on the first page. Copyrights for components of this work owned by others than the author(s) must be honored. Abstracting with credit is permitted. To copy otherwise, or republish, to post on servers or to redistribute to lists, requires prior specific permission and/or a fee. Request permissions from permissions@acm.org.

SenSys'17, November 6-8, 2017, Delft, The Netherlands

(C) 2017 Copyright held by the owner/author(s). Publication rights licensed to the Association for Computing Machinery.

ACM ISBN 978-1-4503-5459-2/17/11 ..\$15.00

https://doi.org/10.1145/3131672.3136956

\section{INTRODUCTION}

IEEE 802.11ah, also known as Wi-Fi HaLow, is a communication standard for heterogeneous Internet of Things (IoT) devices that operate in the unlicensed sub- $1 \mathrm{GHz}$ frequency bands (e.g., the $868 \mathrm{MHz}$ band in Europe) [1]. Its main goal is to provide a good trade-off between range, throughput and energy efficiency. On the MAC layer, several innovative features were introduced, such as fast association and authentication, restricted access window (RAW), traffic indication map (TIM) segmentation and target wake time (TWT). These features allow 802.11ah to support a large amount of energy constrained stations in dense networks and to achieve up to $1 \mathrm{~km}$ range in outdoor environments. The RAW mechanism was introduced in order to address the scalability of a large number of densely deployed devices (up to 8191 supported by one AP). It aims to increase throughput and energy efficiency by dividing stations into different RAW groups, each having access to the channel during a non-overlapping interval [4].

Because of the high data rates that are supported (up to 7.8Mbps), 802.11ah is one of the first IoT technologies in which both highthroughput and low data-rate IoT devices can be supported in an energy efficient manner. In this demonstration we show the ability of IEEE 802.11ah's RAW to provide differentiated Quality of Service (QoS) to densely deployed heterogeneous IoT stations. Specifically, a small set of high-throughput wireless cameras are shown to successfully coexist with a large number of best-effort sensor monitoring stations. The demonstration is performed using an updated version of our ns-3 IEEE 802.11ah implementation [3], adding support for bidirectional traffic and QoS differentiation. Moreover, simulation results are presented in near real-time to the audience using a newly developed visualizer [2].

\section{DEMONSTRATION SCENARIO}

In this demo, we consider a scenario with different devices that have heterogeneous traffic requirements, specifically cameras and sensors. For cameras a consistent reliable throughput is necessary, while the sensors send measurements over specific intervals and have low throughput demands. We show how a proper RAW configuration offers the required differentiated QoS for both types of devices. The main goal of the demo is to show how IEEE 802.11ah is able to manage differentiated devices by way of the RAW mechanism, grouping stations based on their traffic demand in order to 


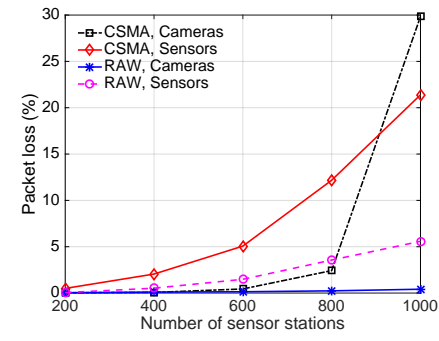

Figure 1: Packet loss comparison for CSMA and RAW, with 8 cameras and a varying number of sensors

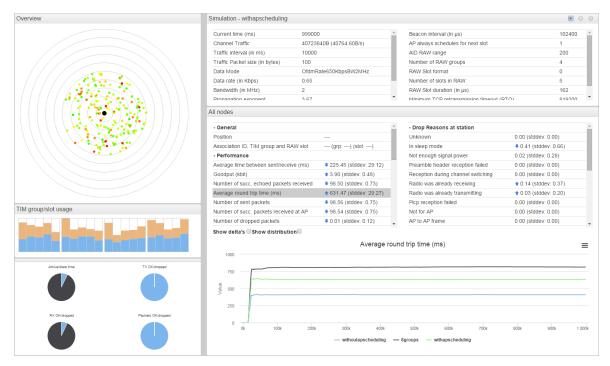

Figure 2: 802.11ah simulation visualizer

Table 1: List of parameters used in the simulation

\begin{tabular}{lr}
\hline Parameter & Value \\
\hline Beacon interval & $102.4 \mathrm{~ms}$ \\
\hline Number of cameras & 8 \\
Camera data rate & $150 \mathrm{kbps}$ \\
Camera MCS & $\mathrm{MCS} 8,2 \mathrm{MHz}$ \\
Camera RAW group duration & $51.2 \mathrm{~ms}$ \\
\hline Number of sensors & $200-1000$ \\
Sensor MCS & $\mathrm{MCS} 0,2 \mathrm{MHz}$ \\
Sensor transmission interval & $10 \mathrm{~s}$ \\
Sensor RAW group duration & $51.2 \mathrm{~ms}$ \\
Sensor RAW slot number per group & 4 \\
Sensor packet size & 64 bytes \\
\hline
\end{tabular}

satisfy heterogeneous throughput, packet loss and delay requirements. It compares RAW to a baseline result using the traditional 802.11 CSMA-CA channel access method.

The QoS differentiation feature is implemented using an intelligent RAW optimization algorithm, based on TAROA [5]. The algorithm splits the stations into two RAW groups, one for highthroughput cameras and one for best-effort sensors. The number of slots per group is dynamically optimized for maximum scalability based on the estimated number of stations in each group. The duration of the two groups is also optimized in real-time in order to minimize latency and packet loss for camera stations, while maintaining a predefined boundary on packet loss for sensor stations.

To illustrate the strength of RAW to provide differentiated QoS to heterogeneous sensors and devices, an example result is shown in Figure 1. This illustrative result is based on a static RAW configuration, as depicted in Table 1 . We consider 8 cameras and a varying number of sensors (i.e., from 200 to 1000) that send traffic to a server via the AP. Two RAW groups are used, the 8 cameras are in one RAW group, all the sensors are in another RAW group which is further split into 4 RAW slots. As shown in Figure 1, CSMA and RAW both almost have no packet loss with 200 sensors. However, as the number of sensors increases to 1000 , sensors get $21 \%$ packet loss for CSMA while only around 5\% packet loss for RAW. With CSMA, the increase of sensors also results in packet loss for cameras, from 600 sensors on, cameras start to suffer packet loss, around $30 \%$ packets are lost with 1000 sensors, while only $0.4 \%$ packet loss occurs when using RAW. With $0.05 \%$ and $5 \%$ packet loss as the upper boundary for cameras and sensors respectively, up to 600 sensors can be supported with CSMA, while 1000 can be supported with RAW. The result clearly reveals that RAW is able to guarantee high performance for a large number of densely deployed IoT stations with heterogeneous traffic requirements.

\section{802.11AH SIMULATION VISUALIZER}

While the ns-3 simulator has several visualization tools, most of these tools only report the final result once the simulation is finished. To analyze the evolution of parameters of interest in near real-time, we have created snapshots of a number of metrics relevant for evaluation of the test network, that are sent to a visualizer which allows easy inspection of the evolution of each metric per station and which reports the overall distribution of the metrics for all stations while the simulation is running. This greatly speeds up the detection of problems in simulations and we can quickly determine if the parameters of the simulation are relevant. It is implemented as a NodeJS web-server which (i) hosts the simulation data sent by ns-3 through TCP connections each second of the simulation time and (ii) forwards the data to the web-browser clients through a websocket in case of live data. The visualizer is shown in Figure 2 and depicts the network topology, RAW slot usage, as well as multiple other metrics for near real-time tracking shown in the tables. Green (good) and red (bad) color gradations in the network topology map depict the behavior of the node in the context of the selected metric.

\section{ACKNOWLEDGMENTS}

Part of this research was funded by the Flemish FWO SBO S004017N IDEAL-IoT (Intelligent DEnse And Longe range IoT networks) project.

\section{REFERENCES}

[1] Evgeny Khorov, Andrey Lyakhov, Alexander Krotov, and Andrey Guschin. 2015. A survey on IEEE 802.11ah: An enabling networking technology for smart cities. Computer Communications 58, May 2014 (2015), 53-69. https://doi.org/10.1016/j. comcom.2014.08.008

[2] Amina Sljivo, Dwight Kerkhove, Ingrid Moerman, Eli de Poorter, and Jeroen Hoebke. 2017. Reliability and scalability evaluation with TCP/IP of IEEE802.11ah networks. Workshop on NS-3 (WNS3) June (2017).

[3] Le Tian, Sebastien Deronne, Steven Latre, and Jeroen Famaey. 2016. Implementation and validation of an IEEE 802.11ah module for NS-3. Workshop on NS-3 (WNS3) 1691, June (2016), 21-27.

[4] Le Tian, Jeroen Famaey, and Steven Latre. 2016. Evaluation of the IEEE 802.11ah Restricted Access Window mechanism for dense IoT networks. WoWMoM 2016 - 17th International Symposium on a World of Wireless, Mobile and Multimedia Networks April (2016). https://doi.org/10.1109/WoWMoM.2016.7523502

[5] Le Tian, Evgeny Khorov, Steven Latré, and Jeroen Famaey. 2017. Real-Time Station Grouping under Dynamic Traffic for IEEE 802.11ah. Sensors 17, 7 (2017). https://doi.org/10.3390/s17071559 